\title{
The effect of vesicourethral anostomosis technique on functional results in retropubic radical prostatectomy
}

\author{
Fuat Kizilay ${ }^{1}$, Serdar Kalemci² ${ }^{2}$ and Adnan Simsir $^{2}$ \\ ${ }^{1}$ Affiliation not available \\ ${ }^{2}$ Ege University Faculty of Medicine
}

March 18, 2021

\begin{abstract}
Aim We aimed to compare the functional results of two different vesicourethral anostomosis (VUA) techniques used in retropubic radical prostatectomy (RRP). Methods A total of 476 patients including the first group with 4 focal VUA at 12-, 3-, 6-, and 9-o'clock positions $(\mathrm{n}=288)$ and the second group with 6 focal VUA at 12-, 2-, 4-, 6-, 8- and 10-o'clock (n = 188) were included in the study. Perioperative data and erectile function and continence status over a 12-month period were compared. Results Demographic and perioperative data were similar between the two groups. The proportion of patients with VUA stricture in the first group was significantly higher than in the second group $(5.1 \%$ vs $3.2 \%, \mathrm{p}=0.017)$. The mean time to stricture development was also shorter in the first group (48.9 vs 74.3 days, $\mathrm{p}=0.002)$. In the second group, the proportion of continent patients at the sixth and twelfth months was higher than the first group $(79.3 \%$ vs $62.8 \%, \mathrm{p}<0.001 ; 92.4 \%$ vs $81.3 \%, \mathrm{p}=0.032$, respectively). There was no significant difference between the two groups in terms of the proportions of potent patients ( $\mathrm{p}=$ 0.194 for 6 months and $\mathrm{p}=0.351$ for 12 months). Conclusions Better continence results can be obtained with the six-focus VUA compared to the four-focus technique. The number of anostomotic sutures in VUA can affect functional results and may be a decisive factor for surgeons who focus on functional results as well as oncological results.
\end{abstract}

The effect of vesicourethral anostomosis technique on functional results in retropubic radical prostatectomy

\section{Aim}

We aimed to compare the functional results of two different vesicourethral anostomosis (VUA) techniques used in retropubic radical prostatectomy (RRP).

\section{Methods}

A total of 476 patients including the first group with 4 focal VUA at 12-, 3-, 6-, and 9-o'clock positions (n $=288)$ and the second group with 6 focal VUA at 12-, 2-, 4-, 6-, 8- and 10-o'clock $(\mathrm{n}=188)$ were included in the study. Perioperative data and erectile function and continence status over a 12 -month period were compared.

\section{Results}

Demographic and perioperative data were similar between the two groups. The proportion of patients with VUA stricture in the first group was significantly higher than in the second group $(5.1 \%$ vs $3.2 \%, \mathrm{p}=0.017)$. The mean time to stricture development was also shorter in the first group (48.9 vs 74.3 days, $\mathrm{p}=0.002)$. In the second group, the proportion of continent patients at the sixth and twelfth months was higher than the first group ( $79.3 \%$ vs $62.8 \%, \mathrm{p}<0.001 ; 92.4 \%$ vs $81.3 \%, \mathrm{p}=0.032$, respectively). There was no significant difference between the two groups in terms of the proportions of potent patients $(\mathrm{p}=0.194$ for 6 months and $\mathrm{p}=0.351$ for 12 months). 


\section{Conclusions}

Better continence results can be obtained with the six-focus VUA compared to the four-focus technique. The number of anostomotic sutures in VUA can affect functional results and may be a decisive factor for surgeons who focus on functional results as well as oncological results.

Key words: prostate cancer, retropubic radical prostatectomy, functional outcomes, vesicourethral anostomosis, incontinence.

\section{Introduction}

Prostate cancer (PCa), the most common cancer of men, accounts for approximately one third of all cancers in men. ${ }^{1}$ Although there are treatment options such as high-intensity focused ultrasound, cryoablation and radiotherapy, the vast majority of men undergo open retropubic radical prostatectomy (RRP), which is the gold standard in the treatment of this disease. ${ }^{2}$ With the developing technique and technology, minimally invasive treatment methods have become popularized in urological oncological surgery. Robotic radical prostatectomy, which is one of these techniques, has revolutionized PCa surgical treatment with the benefits it provides to surgeons. However, the high cost of the robotic platform and the long learning curve of the laparoscopic technique prevented them from spreading globally, and in most centers, RRP is still the most performed technique.

In radical prostatectomy, it is of great importance to achieve optimal functional results in addition to oncological control. Vesicourethral anostomosis (VUA) technique affects significant complications such as colllum sclerosis and incontinence affecting the course of surgery results. Various modifications have been developed to optimize anostomosis and avoid those complications. In the past, Walsh, who has contributed greatly to the development of the RRP technique, proposed 6 focal sutures for VUA. ${ }^{3}$ Then, this technique was tried to be modified and the results of studies dealing with suturing sites, numbers and continuous suturing were published. ${ }^{4-6}$ These techniques have been tried to be performed in laparoscopic and robotic techniques besides open technique. In addition to these modifications, a technique, in which a completely sutureless anostomosis was made, appeared in the literatüre. ${ }^{7}$ Their common goal was to optimize the functional results after VUA.

It is an undeniable fact that VUA technique affects important functional results, especially anostomosis stricture. There is no consensus in the literature on the optimal anostomosis technique and more studies are needed to clarify this issue. In this study, we aimed to evaluate the effect of two different VUA techniques that we frequently use in our clinic on functional outcomes.

\section{Materials and Methods}

\section{Patient selection and data collection}

Five hundred thirty-five patients who underwent RRP between June 2015 and June 2019 were evaluated. Patients who had a history of pelvic radiotherapy, urethral intervention, overactive bladder and / or neurogenous bladder and who used drugs that could affect continence were excluded from the study. The remaining 476 patients were included in the study. Patients were divided into two groups according to the VUA technique: patients who underwent four focal VUA (12-, 3-, 6-, and 9-o'clock positions) (group I) and patients who underwent six focal VUA (12-, 2-, 4-, 6-, 8- and 10-o'clock positions) (group II). There were 288 patients in group I and 188 patients in group II. The flow chart of the study is shown in Figure 1 . All data were collected retrospectively from the hospital database. Demographic, perioperative and postoperative data and functional results of the two groups were recorded and analyzed.

\section{Evaluation criteria}

Patients who were not using pads or using a single pad for protection within 24 hours were considered as continent. Erectile function was evaluated using the International Erectile Function Index (IIEF) and patients with IIEF scores below 10 were considered to have erectile dysfunction. ${ }^{8}$ Patients who were followed for at least 12 months after surgery were included in the study. Patients suffering from urinary difficulty 
in the postoperative period were evaluated for VUA stricture. In patients with suspected stricture in the uroflowmetry test, a definitive diagnosis was made by performing urethroscopy.

Retropubic radical prostatectomy technique

The RRP was carried out in accordance with the technique described by Walsh in $1988 .{ }^{9}$ Under general intratracheal anesthesia, an 18 Fr Foley catheter was introduced and the skin, subcutaneous, and muscles were cut through the suprapubic incision. Then the endopelvic fascia was opened, the dorsal venous plexus was ligated with 0 Vicryl suture and cut. The urethra was released, suspended and cut. The prostate was released by sharp and blunt dissections from the denonvilliers fascia. Bilateral ductus deferens were clamped and cut, the right and left seminal vesicles were removed enblock with the prostate. A 22 Fr Foley catheter was introduced. VUA was performed from 4 or 6 foci with $2 / 0$ monocryl sutures. One Jackson-pratt ${ }^{\circledR}$ drain was inserted into the surgical area, fasia was closed with 0 PDS suture, and skin with stapler, respectively. In the four focus technique, sutures were placed at the 12-, 3-, 6-, and 9-o'clock positions. In the six focus technique, sutures were placed at 12-, 2-, 4-, 6-, 8- and 10-o'clock positions. VUA techniques with four and six focus are schematized in Figure 2 .

Outcome measures

The primary endpoint of the study was the evaluation of the relationship between the vesicourethral anostomosis technique and elapsed time for causing collum sclerosis, and the number of recurrences. Analysis of the effect of the technique on erectile function and continence results was the secondary endpoint.

Statistical analysis

While Student's t-test was used for the analysis of quantitative data, chi-square test was used for the analysis of categorical data. P values less than 0.05 were considered statistically significant. SPSS 23.0 for Macintosh was used for all analyzes.

\section{Results}

The data of 476 patients whose information was obtained and who underwent RRP were evaluated. The demographic data and basal PSA values of the first group with four-focus VUA and the second group with six-focus VUA were similar. The majority of the patients in both groups consisted of patients with Gleason score $3+3$ and $3+4$. Prostate volume and perioperative data were also similar between the two groups. There was no significant difference between the $\mathrm{T}$ stage of the prostatectomy specimens. Comparison of the demographic and perioperative data of the groups is summarized in Table 1 .

The proportion of patients with VUA stricture in the first group was significantly higher than in the second group $(5.1 \%$ vs $3.2 \%, \mathrm{p}=0.017)$. Also, the mean time to stricture development was shorter in the first group (48.9 vs 74.3 days, $\mathrm{p}=0.002$ ). Although the continence rates in the sixth month decreased in both groups compared to the preoperative condition, the continence rate in the second group was higher than the first group $(79.3 \%$ vs $62.8 \%, \mathrm{p}<0.001)$. At 12 months, improvement in continence status was observed in both groups, but the second group had a higher rate of continence again $(92.4 \% \mathrm{vs} 81.3 \%, \mathrm{p}=0.032)$. Continence restoration curves for the two groups are shown in Figure $\mathbf{3}$. The proportions of potent patients in the two groups tended to decrease at 6 months, but improvement was seen at 12 months. In addition, there was no significant difference between the two groups in terms of potent patient rates $(\mathrm{p}=0.194$ for the 6 th month and $\mathrm{p}=0.351$ for the 12 th month). The restoration curves comparing the potency rates of the two groups are schematized in Figure 4. The comparison of the functional results of the two groups at 6 and 12 months is summarized in Table 2 .

\section{Discussion}

In patients undergoing RRP for $\mathrm{PCa}$, it is critical to provide optimal functional results as well as oncological control. The most important of these are results related to continence and erectile function. Although these results have been tried to be optimized in recent years with the robotic platform that enables large and 
three-dimensional images, the open retropubic technique still continues to be performed at a considerable rate in the world. ${ }^{10}$

There is a consensus that the VUA technique is particularly important in maintaining postoperative continence. ${ }^{11}$ In this study, in which we analyzed the effect of these techniques on functional results in patients who underwent two different VUA techniques in our clinic, we revealed that although the continence results were better in the 6-focal anostomosis group compared to the 4 -focus group, it did not have a significant effect on erectile function results. Our study differs from previous studies by being the first to compare 4- and 6-focus VUA techniques head-to-head.

In a randomized controlled study in which the two techniques used in our study were compared with the anostomosis technique performed with 2 U sutures at 6 and 12 o'clock alignment, the number of sutures did not affect the perioperative and postoperative parameters, but the VUA duration was significantly shorter in patients operated with the 2 -suture technique compared to the others ${ }^{5}$. This study is important as it is the first prospective study to focus on the VUA technique during RRP. In our study, the duration of VUA was not available, but the total operation times were similar between the two groups. However, the conclusion, that the number of sutures did not affect the continence results revealed in the previous study, was in favor of the group in which the number of sutures was high in our study. The factors that predispose to the development of VUA are not fully understood. Anastomotic technique, previous endoscopic prostate surgery, failure to obtain a watertight anastomosis resulting in urine extravasation, excessive blood loss and excessive diathermy for hemostasis of the bladder neck are the main culprits. ${ }^{12}$ We hypothesize that the water-tight anostomosis is more safely achieved in the six-focal anostomosis because the suture line is denser and closer than the 4-focal technique, and that better continence results are obtained as a result. As a result of VUA performed with more frequent suturing, it is possible to expect that foreign tissue penetrates the anostomosis line less and thus mucosa-mucosa convergence is healthier. In a meta-analysis in which a total of 9 studies including 1475 patients were evaluated, continuous suturing and intermittent suturing were compared, and although continuous suturing provided shorter catheterization time, anastomosis time and a lower rate of extravasation, no significant difference was found between continence rates and urethral stricture rates in the month was 3,6 , and 12 and the duration of hospital stay between the two techniques. It was underlined that the suture technique should be chosen according to the surgeon's experience and technical approach. ${ }^{13}$ In another retrospective analysis, the results of sutureless anostomosis in patients undergoing open and laparoscopic radical prostatectomy were evaluated. In this VUA technique, which is designed completely without sutures, the catheter balloon was inflated and traction was applied. The authors observed a significant rate of stricture (13.6\%) while maintaining around $90 \%$ continence rates in patients. ${ }^{7}$ Although this rate underlines the importance of sutured VUA, uncertainties remain as to which VUA technique is superior. Although the general opinion in the literature is that the number of sutures does not affect the risk of VUA and continence outcomes, there is consensus that a water-tight anostomosis is vital to prevent urine extravasation. ${ }^{14,15}$

During the operation, excessive blood loss and / or hematoma formation may contribute to the development of VUA by negatively affecting tissue healing by causing fibrosis and scar formation in the surrounding tissue. The fact that the mean blood loss is similar in both groups reveals that the effect of this factor is minimal in our patients. VUA stricture development is an independent risk factor for the development of postoperative incontinence. ${ }^{15}$ On the other hand, prevention of anostomotic strictures is one of the most important factors for continence recovery and also shortens the time required for continence recovery. ${ }^{16}$ In our study, in the first group with a higher stricture rate, the continence rates were found to be lower in proportion to this outcome.

The application of oxidized cellulose sponges around the anastomotic site can be used to block exudates and materials such as fluid or cellular debris escaping from blood vessels. If urethral exudates accumulate in the urethral cavity, it can lead to inflammation or even infection of the urethra, jeopardizing healing. ${ }^{17}$ The study using these materials provided slightly lower long-term incontinence rates than ours. ${ }^{5}$ Cellulose sponges can be effective agents in maintaining continence function. Some modifications are recommended for use in VUA 
for optimum postprostatectomic erectile function. One of these is that after dissecting the anterior half of the urethra, four pre-placed sutures are placed between 3 and 6 o'clock, and anostomotic sutures are placed on the posterior half after the neurovascular bundle is separated from the prostate apex. ${ }^{3,18}$ Most of the patients in our group consisted of patients with tumors limited to the prostate, and we performed nervesparing prostatectomy in these patients. At the end of a one-year follow-up, we achieved approximately $80 \%$ of erectile function restoration and we concluded that the two VUA techniques we used did not have a significant effect on erectile functions. For the preservation of erectile function, the application of neuroprotective surgery is more important than the VUA technique according to the risk assessment on patient basis.

In our study, we found that, contrary to the literature, the VUA technique affected both stricture development time and stricture rate. We did not evaluate the preoperative voiding functions of the patients with objective questionnaires. Not knowing the patients' basal functions can create confusion in comparing final functions, but preoperative contience is not the only factor affecting this. Other reported risk factors include patient age, disease stage, surgical technique, surgeon's experience, and previous endoscopic prostate surgery, and there was no significant difference in these factors between the groups. ${ }^{18}$

Our study has some important limitations. Since we do not know the preoperative continence and erectile functions of the patients, we could not analyze how much they changed according to basal levels in the postoperative period. Surgery performed by different surgeons may cause a surgeon selection bias, but this may be more consistent in that it is a real-life picture of the situation in most reference centers. Evaluating continence status with questions such as present / absent instead of validated questionnaire forms might have overshadowed the objective evaluation. On the other hand, in many studies in the literature this function has been evaluated in this way. The main strength of our study is that it is the first study to compare the functional results of 4- and 6-focus VUA techniques, and obtaining 12-month restoration curves specific to the two functions appears to be a valuable finding.

As a result, we found better continence results with 6-focus VUA than with the 4-focus technique. There was no significant difference between the two techniques in terms of other postoperative complications and erectile function. Contrary to the general belief in the literature, the number of anostomotic sutures in VUA may affect functional results and may be decisive for surgeons who focus on functional results as well as oncological results. Although the most important determining factor is the surgeon's habits and experience, the 6-focus VUA technique we schematized in our study can be preferred due to its easy application and similar operation time with the other technique. More consistent results can be expected with prospective, randomized, controlled studies with a higher number of cases.

\section{References}

1. Siegel RL, Miller KD, Jemal A. Cancer statistics, 2019. CA: a cancer journal for clinicians . Jan 2019;69(1):7-34. doi:10.3322/caac.21551

2. Sommers BD, Beard CJ, D'Amico AV, Kaplan I, Richie JP, Zeckhauser RJ. Predictors of patient preferences and treatment choices for localized prostate cancer. Cancer . Oct 15 2008;113(8):2058-67. doi: $10.1002 /$ cncr. 23807

3. Walsh PC. Anatomic radical prostatectomy: evolution of the surgical technique. J Urol . Dec 1998;160(6 Pt 2):2418-24. doi:10.1097/00005392-199812020-00010

4. Mazaris EM, Chatzidarellis E, Varkarakis IM, Dellis A, Deliveliotis C. Reducing the number of sutures for vesicourethral anastomosis in radical retropubic prostatectomy. International braz $j$ urol : official journal of the Brazilian Society of Urology . Mar-Apr 2009;35(2):158-63. doi:10.1590/s1677-55382009000200005

5. Gallo L, Perdona S, Autorino R, et al. Vesicourethral anastomosis during radical retropubic prostatectomy: does the number of sutures matter? Urology . Mar 2007;69(3):547-51. doi:10.1016/j.urology.2006.12.016

6. Van Velthoven RF, Ahlering TE, Peltier A, Skarecky DW, Clayman RV. Technique for laparoscopic running urethrovesical anastomosis:the single knot method. Urology. Apr 2003;61(4):699-702. doi:10.1016/s0090- 
4295(02)02543-8

7. Simforoosh N, Javaherforooshzadeh A, Aminsharifi A, Tabibi A. Early continence after open and laparoscopic radical prostatectomy with sutureless vesicourethral alignment: an alternative technique, 8 years' experience. Urology journal . Summer 2009;6(3):163-9.

8. Akkus E, Kadioglu A, Esen A, et al. Prevalence and correlates of erectile dysfunction in Turkey: a population-based study. Eur Urol . Mar 2002;41(3):298-304. doi:10.1016/s0302-2838(02)00027-1

9. Walsh PC. Nerve sparing radical prostatectomy for early stage prostate cancer. Seminars in oncology . Aug 1988;15(4):351-8.

10. Cooperberg MR, Broering JM, Carroll PR. Time trends and local variation in primary treatment of localized prostate cancer.Journal of clinical oncology : official journal of the American Society of Clinical Oncology . Mar 1 2010;28(7):1117-23. doi:10.1200/JCO.2009.26.0133

11. Lee SW, Han DH, Lee KS, Jeon SS. Effect of Continuous Urethro-Vesical Anastomosis Technique in Incontinence After Radical Retropubic Prostatectomy, 1:1 Matching Study. International neurourology journal . Jun 2015;19(2):113-9. doi:10.5213/inj.2015.19.2.113

12. Besarani D, Amoroso P, Kirby R. Bladder neck contracture after radical retropubic prostatectomy. BJU international . Dec 2004;94(9):1245-7. doi:10.1111/j.1464-410X.2004.05151.x

13. Kowalewski KF, Tapking C, Hetjens S, et al. Interrupted versus Continuous Suturing for Vesicourethral Anastomosis During Radical Prostatectomy: A Systematic Review and Meta-analysis. European urology focus . Nov 2019;5(6):980-991. doi:10.1016/j.euf.2018.05.009

14. Klein EA. Modified apical dissection for early continence after radical prostatectomy. The Prostate . 1993;22(3):217-23. doi:10.1002/pros.2990220304

15. Licht MR, Klein EA, Tuason L, Levin H. Impact of bladder neck preservation during radical prostatectomy on continence and cancer control. Urology . Dec 1994;44(6):883-7. doi:10.1016/s0090-4295(94)80175-4

16. Kaye KW, Creed KE, Wilson GJ, D'Antuono M, Dawkins HJ. Urinary continence after radical retropubic prostatectomy. Analysis and synthesis of contributing factors: a unified concept. British journal of urology . Sep 1997;80(3):444-501. doi:10.1046/j.1464-410x.1997.00373.x

17. Lawrentschuk N, Bolton DM, Angus D. Fenestrated urethral catheter to aid anastomotic drainage after radical prostatectomy. Urology . Jan 2005;65(1):160-2. doi:10.1016/j.urology.2004.08.029

18. Eastham JA, Kattan MW, Rogers E, et al. Risk factors for urinary incontinence after radical prostatectomy. J Urol . Nov 1996;156(5):1707-13.

Figure legends

Figure 1. Flow-chart for the study population

Figure 2. Schematicization of anostomosis sutures and numbers in vesicourethral anostomosis region. A) Four-focus anastomosis technique, B) Six-focus anastomosis technique

Figure 3. Kaplan-Meyers curves for the continence restoration in the studied patient groups (Group I: four focal vesicourethral anostomosis anostomosis, Group II: six focal vesicourethral anostomosis)

Figure 4. Kaplan-Meyers curves for the potency restoration in the studied patient groups (Group I: four focal vesicourethral anostomosis anostomosis, Group II: six focal vesicourethral anostomosis)

Table 1. Comparison of demographic and peripoerative data of the two groups 


\begin{tabular}{llll}
\hline & $\begin{array}{l}\text { Group I (four sutured } \\
\text { anastomosis) }\end{array}$ & $\begin{array}{l}\text { Group II (six sutured } \\
\text { anastomosis) }\end{array}$ & P value \\
\hline Number of patients & 288 & 188 & \\
Age & $56.8 \pm 12.9$ & $61.3 \pm 16.6$ & 0.562 \\
BMI & $22.4 \pm 9.1$ & $21.8 \pm 8.7$ & 0.408 \\
PSA (ng/mL) & $8.2 \pm 1.1$ & $7.4 \pm 0.6$ & 0.255 \\
Gleason score 3+3 3+4 & $97(33.7 \%) 116(40.3 \%)$ & $60(31.9 \%) 78(41.5 \%) 33$ & 0.621 \\
$4+34+4$ 4+5 & $56(19.4 \%) 12(4.2 \%) 7$ & $(17.5 \%) 9(4.8 \%) 8$ & \\
& $(2.4 \%)$ & $(4.3 \%)$ & 0.112 \\
Prostate volume (mL) & $42.2 \pm 18.3$ & $37.5 \pm 14.1$ & 0.398 \\
Operative time (min) & $112 \pm 29$ & $126 \pm 33$ & 0.702 \\
$\begin{array}{l}\text { Estimated blood loss } \\
\text { (ml) }\end{array}$ & $68 \pm 16$ & $74 \pm 21$ & 0.081 \\
Drainage amount (ml) & $42 \pm 16$ & $36 \pm 12$ & 0.206 \\
Length of & $2.3 \pm 0.8$ & $2.5 \pm 0.9$ & \\
hospitalization (day) & & & 0.190 \\
$\begin{array}{l}\text { Blood transfusion } \\
\text { (unit) }\end{array}$ & $0.6 \pm 0.2$ & $0.8 \pm 0.4$ & \\
Foley duration (day) & $8.7 \pm 1.1$ & $9.1 \pm 1.3$ & 0.836 \\
Pathologic stage T1 T2 & $72(25 \%) 114(39.6 \%) 102$ & $48(25.5 \%) 96(51.1 \%)$ & 0.070 \\
T3 & $(35.4 \%)$ & $(23.4 \%)$ & \\
\hline
\end{tabular}

Values are given as mean \pm standard deviation or number (percent), PSA: prostate-spesific antigen, BMI: body mass index

Table 2. Comparison of functional results of two groups

\begin{tabular}{llll}
\hline & Group I (four sutured anastomosis) & Group II (six sutured anastomosis) & P value \\
\hline VUA stricture & $15(5.1 \%)$ & $6(3.2 \%)$ & 0.017 \\
Time to stricture (day) & $48.9 \pm 16.6$ & $74.3 \pm 21.2$ & 0.002 \\
Continence rate at 6 month & $181(62.8 \%)$ & $149(79.3 \%)$ & $<0.001$ \\
Continence rate at 12 month & $234(81.3 \%)$ & $174(92.4 \%)$ & 0.032 \\
Potency rate at 6 month & $206(71.5 \%)$ & $141(74.8 \%)$ & 0.194 \\
Potency rate at 12 month & $226(78.6 \%)$ & $153(81.5 \%)$ & 0.351 \\
\hline
\end{tabular}

Values are given as mean \pm standard deviation or number (percent), VUA: vesicourethral anostomosis 

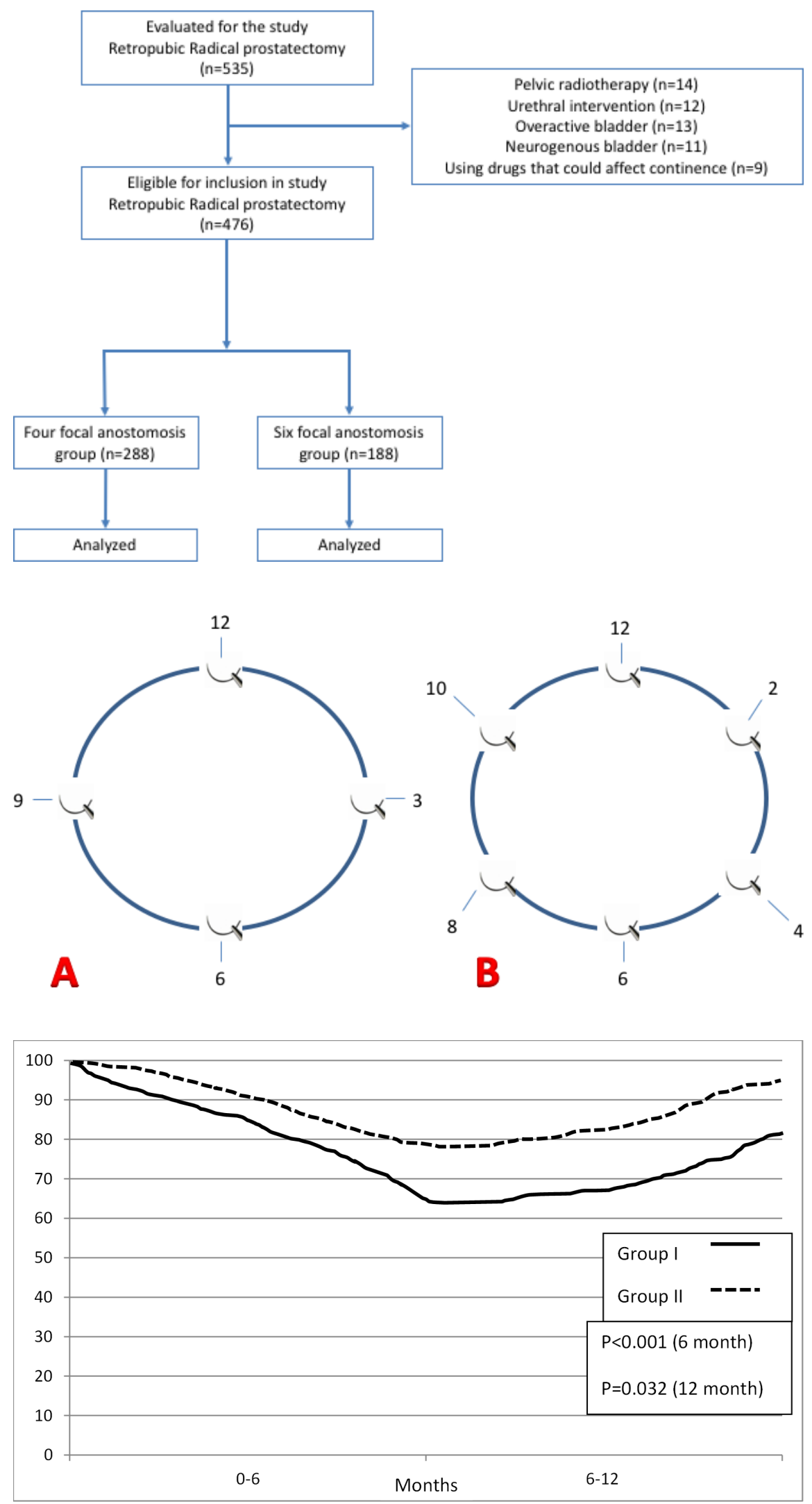


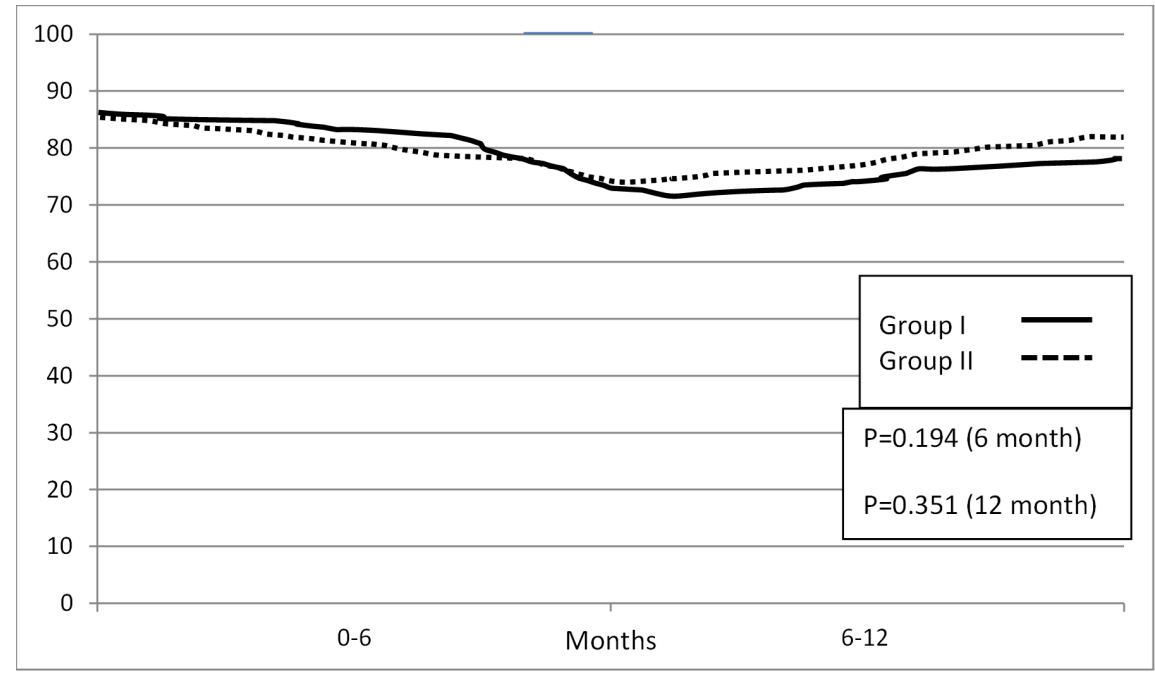

